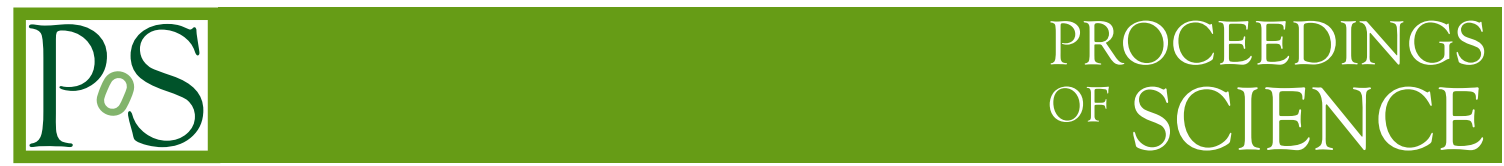

\title{
Vector boson associated with jets in CMS
}

\section{Qun Wang*for the CMS collaboration}

Deutsches Elektronen-Synchrotron, D-22607 Hamburg, Germany

E-mail: qun.wang@desy.de

The study of the associated production of vector bosons and jets constitutes an excellent enviroment to check numerous QCD predictions. Total and differential cross sections of vector bosons produced in association with jets have been studied in pp collisions using CMS data. Differential distributions as function of a broad range of kinematical observables are measured and compared with theoretical predictions.

*** The European Physical Society Conference on High Energy Physics (EPS-HEP2021), *** *** 26-30 July $2021 * * *$

*** Online conference, jointly organized by Universität Hamburg and the research center DESY ***

\footnotetext{
${ }^{*}$ Speaker
} 
The production of Z-bosons in Drell-Yan (DY) [1] in pp collisions is one of the most precisely measured processes at the LHC [2-6]. The measurement of vector boson production in association with jets provides a sensitive evaluation of the accuracy of QCD modeling, which is important for modeling the production mechanism involved in the Higgs boson and new physics searches. The production of a vector boson together with jets is a dominant background in many Standard Model (SM) processes, such as Higgs production, $t \bar{t}$ production for searches for dark matter and supersymmetric particles. Comparisons of measurements with the predictions from Monte Carlo (MC) based event generators and higher order theoretical calculations improves our understanding of the prediction uncertainties and motivates the development of generators.

\section{1. $\mathrm{Z}$ boson invisible width at $13 \mathrm{TeV}$}

A direct measurement of the invisible width of the $\mathrm{Z}$ boson [7] in proton-proton collisions at a center-of-mass energy of $13 \mathrm{TeV}$ is presented, based on data recorded by the CMS experiment [8] corresponding to an integrated luminosity of $36.3 \mathrm{fb}^{-1}$. The $\mathrm{Z}$ boson invisible width is extracted from the ratio of measured cross sections of the $\mathrm{Z}$ boson to neutrinos and to the charged dileptons.

In the analysis, the events can be categorized into three main regions: the $p_{\mathrm{T}}^{\text {miss }}$ plus jets (where $p_{\mathrm{T}}^{\text {miss }}$ is the missing transverse momentum vector) signal region that is composed predominantly of $\mathrm{Z} \rightarrow v \bar{v}$ events, a dilepton plus jets region for $\mathrm{Z} / \gamma^{*} \rightarrow \mu \mu$ and $\mathrm{Z} / \gamma^{*} \rightarrow e e$ determination, and a single lepton plus jets region, $l+$ jets, where $l=e, \mu, \tau_{\mathrm{h}}$ (hadronically decaying $\tau$ ) for $\mathrm{W}+$ jets measurement.

A set of baseline selection criteria is applied to all regions, requiring $p_{\mathrm{T}}^{\text {miss }}>200 \mathrm{GeV}$ and the leading jet to have $p_{\mathrm{T}}>200 \mathrm{GeV}$. The value of $\left|p_{\mathrm{T}, \text { Calo }}^{\text {miss }}-p_{\mathrm{T}}^{\text {miss }}\right| / p_{\mathrm{T}}^{\text {miss }}$ is required to be less than 0.5 , where $p_{\mathrm{T} \text {,Calo }}^{\text {miss }}$ is the $p_{\mathrm{T}}^{\text {miss }}$ measured only by the calorimeter systems and corrected for the presence of leptons. Events are vetoed if they contain jets with $p_{\mathrm{T}}>40 \mathrm{GeV}$ and $|\eta|>2.4$; there is an isolated photon passing a loose identification criteria with $p_{\mathrm{T}}>25 \mathrm{GeV}$ and $|\eta|<2.5$; they contain a b-tagged jet with $p_{\mathrm{T}}>40 \mathrm{GeV}$ and $|\eta|<2.4$.

Many background processes have been considered in the analysis, such as $\mathrm{W}+\mathrm{jets}, \gamma+\mathrm{jets}$, $t \bar{t}+$ jets, diboson, QCD multijet and single top. Background is suppressed by vetoing events containing an isolated muon or electron with $p_{\mathrm{T}}>10 \mathrm{GeV}$ and $|\eta|<2.4$, or a $\tau_{\mathrm{h}}$ with $p_{\mathrm{T}}>20 \mathrm{GeV}$ and $|\eta|<2.3$. The largest background, $\mathrm{W}+$ jets, is estimated from data using the control region of $l+$ jets. A transfer factor has been determined as a function of $p_{\mathrm{T}}^{\mathrm{miss}}$ and used to correct the event yields in data to obtain the expected number of $\mathrm{W}+$ jets events in the signal region. The contribution from QCD multijet events is estimated from a control region. Other backgrounds are taken from simulation.

The invisible width of the $\mathrm{Z}$ boson, $\Gamma_{\mathrm{inv}}$, is extracted from a simultaneous likelihood fit to data in the $p_{\mathrm{T}}^{\text {miss }}$ plus jets, $\mathrm{Z} / \gamma^{*}(\mu \mu$ and ee) plus jets, $\mu+$ jets, and $\mathrm{e}+$ jets regions. The observed $\mathrm{Z}$ boson invisible width is measured to be $\Gamma_{\text {inv }}=523 \pm 3$ (stat) \pm 16 (syst) $\mathrm{MeV}$. Figure 1 shows a comparison with the direct measurements from the LEP experiments L3, OPAL and ALEPH and the LEP combined measurement. The CMS measurement of the $\mathrm{Z}$ boson invisible width with an uncertainty of $16 \mathrm{MeV}$ is comparable to the LEP combined result. and is the single most precise direct measurement of $\Gamma_{\text {inv }}$. 


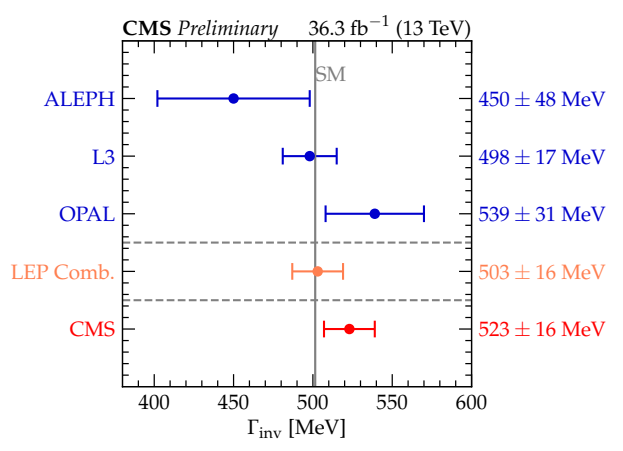

Figure 1: Measurements of the $\mathrm{Z}$ boson invisible width with the CMS experiment and the results by the LEP experiments [7].

\section{Multi-differential $\mathrm{Z}+$ jets cross sections at $13 \mathrm{TeV}$}

The cross sections for the production of $\mathrm{Z}$ bosons in association with jets, in proton-proton collision at a center-of-mass of $13 \mathrm{TeV}$ [9] is performed with data recorded in 2016 by CMS, corresponding to $35.9 \mathrm{fb}^{-1}$ of integrated luminosity. The cross section has been measured as a function of jet multiplicity $\left(N_{\text {jets }}\right)$ and the individual jet kinematic variables, and the scalar sum of the jet transverse momenta $\left(H_{\mathrm{T}}\right)$ for events up to 5 energetic jets.

The measured $Z+j e t s$ differential cross sections is compared to three predictions. The first one is MadGraph5_AMC@NLO (denoted MG5_AMC) including NLO Matrix Elements (MEs) calculations for $\mathrm{pp} \rightarrow \mathrm{Z}+$ Njets with $\mathrm{N}$ up to 2 . PYтHIA 8 is used to model the parton showers and handronization, the merging of PYтнIA 8 and MEs is performed with the FxFX scheme. The second one is MG5_AMC interfaced with PYTHIA 8, with MEs computed at Leading Order (LO) for five processes $\mathrm{pp} \rightarrow \mathrm{Z}+$ Njets, $\mathrm{N}=0 \ldots 4$ and matched to the parton shower using $k_{T}-$ MLM scheme. The CUETP8M1 PYthia 8 tune is used for both MG5_AMC samples. The third calcualtion uses the Geneva 1.0-RC3 MC program, where an NNLO calculation for Drell-Yan (DY) production is combined with higher-order resummation. Logarithms of the 0 -jettiness resolution variable, $\tau$, also known as beam trust, are resummed at NNLL'. It is interfaced with PYTHIA 8 using the CUETP8M1 tune.

The measured cross section are unfolded to the particle level. The phase space is restricted to events with two leptons with $|\eta|<2.4$, $p_{\mathrm{T}}^{\text {leading lep }}>25 \mathrm{GeV}$, $p_{\mathrm{T}}^{\text {sub-leading }}>20 \mathrm{GeV}$, and a $\mathrm{Z}$ boson mass between 71 and $111 \mathrm{GeV}$. Jets are required to have $p_{\mathrm{T}}>30 \mathrm{GeV},|\eta|<2.4$ and a spatial separation from the dressed leptons of $\Delta R>0.4$.

The measured cross sections as a function of the inclusive jet multiplicity are shown in fig 2 . The agreement is very satisfactory for the distributions for all the theoretical estimations up to the maximum number of final state partons included in the ME in the MC generators. The Geneva generator predicts a much steeper spectrum than observed due to the lack of hard jets at ME level beyond two. The double differential cross sections as a function of leading jet $p_{\mathrm{T}}$ is shown in fig 2 . In general, all the predictions are in the agreement with data and the NLO prediction provides a better description than LO MG5_AMC and GENEva for double differential cross sections. 

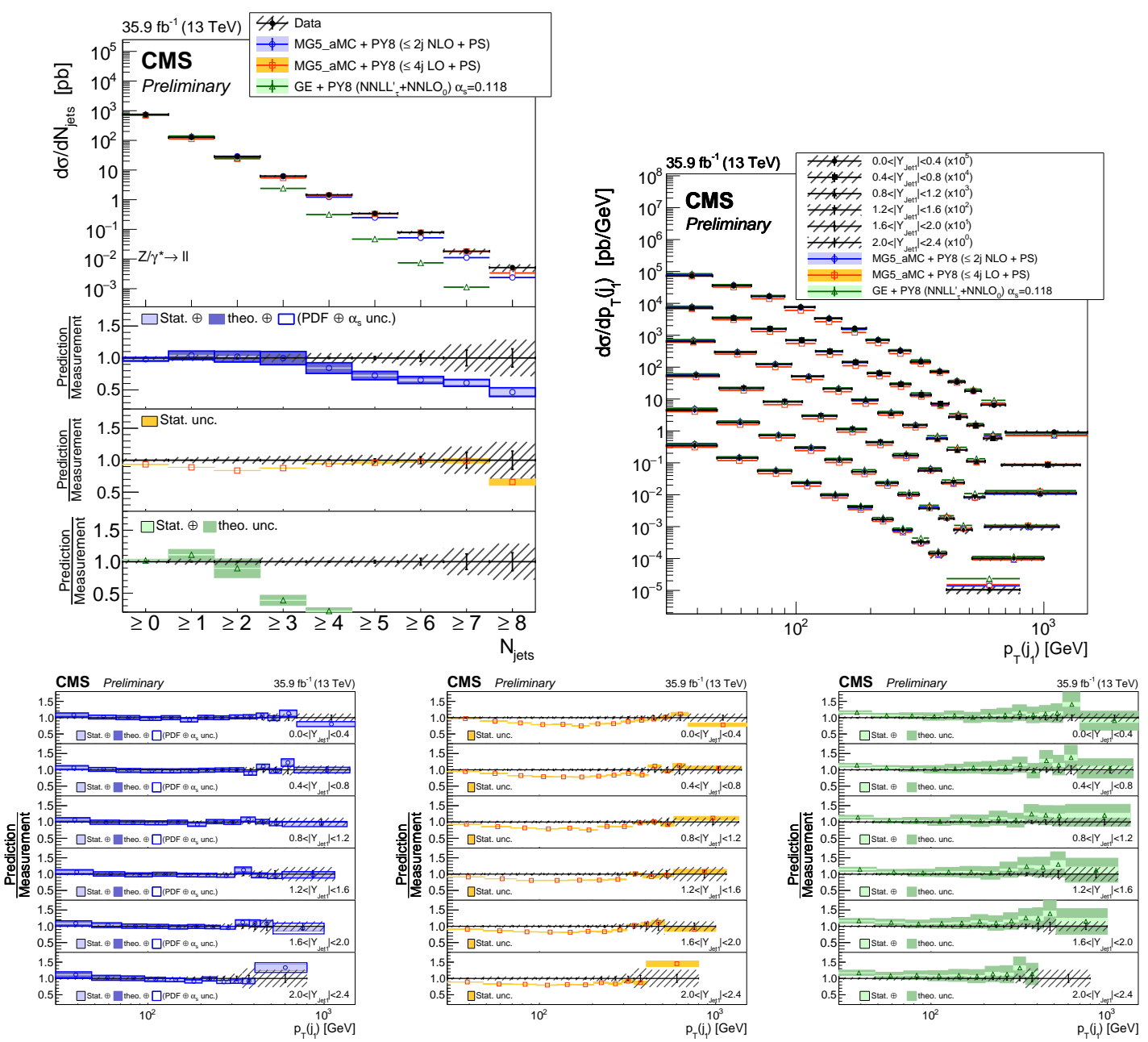

Figure 2: Measured Z+jets differential cross section as a function of the inclusive jet multiplicity (top left), and the double differential cross section as a function of the leading jet $p_{\mathrm{T}}$ (top right) [9]. The measurement is compared to NLO MG5_AMC (bottom left), LO MG5_AMC (bottom middle), and Geneva (bottom right).

\section{Azimuthal correlations in $\mathrm{Z}+$ jets at $\mathbf{1 3} \mathrm{TeV}$}

We study in detail the production of a $\mathrm{Z}$ boson with associated jets at a center-of-mass energy of $13 \mathrm{TeV}$ with data recorded at the LHC corresponding to a luminosity of $36.3 \mathrm{fb}^{-1}$ [10]. We measure the jet multiplicity, the azimuthal correlation between the $\mathrm{Z}$ boson and the leading jet $\Delta \phi_{\mathrm{Z}, \text { Jet } 1}$, as well as the correlation between the two leading jets $\Delta \phi\left(j_{1}, j_{2}\right)$ in different regions of $p_{\mathrm{T}}(\mathrm{Z})$.

The measured differential cross sections are compared to four predictions. The prediction MG5_AMC interfaced PYTHIA 8 at NLO and the NNLO calculation GeNEVA have been described in the previous section. Two predictions are obtained from MG5_AMC with Parton Branching (PB) transverse momentum dependent parton densities (TMDs) and parton shower as implemented in CASCADE3. The matrix elements are calculated at NLO for $Z+1$ and $Z+2$ partons (labelled as MG5AMC+CA3 $(Z+1) \mathrm{NLO}$ and MG5AMC+CA3 $(Z+2) \mathrm{NLO})$ separately.

The phase space for the particle level is restricted to events with a $Z$ boson mass between 76 

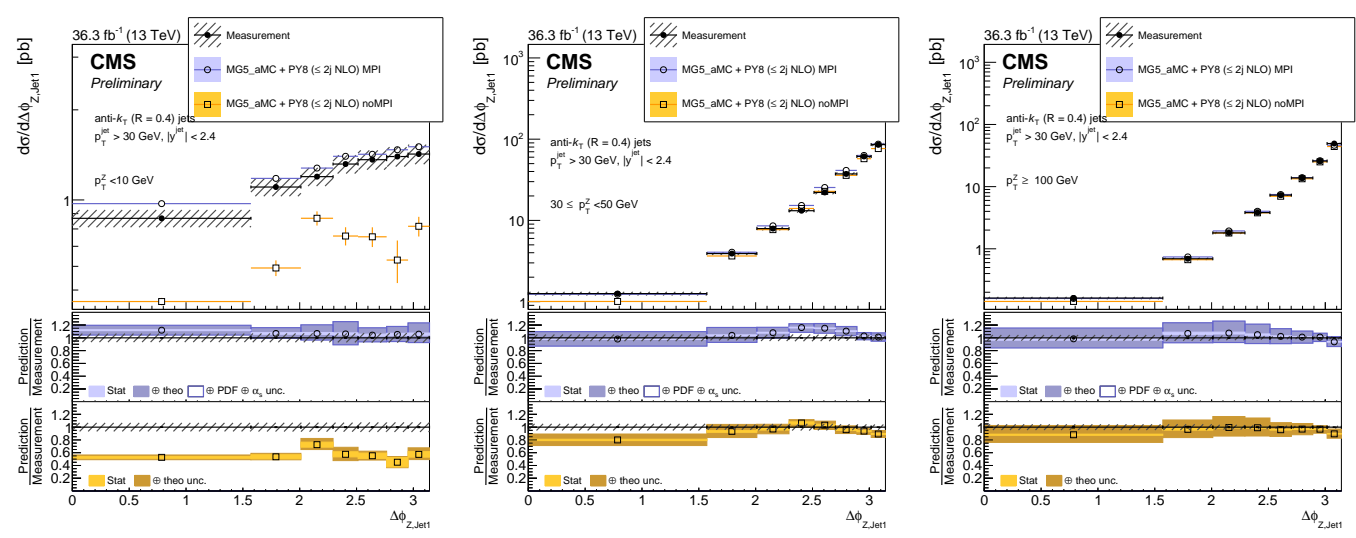

Figure 3: Measured $\mathrm{Z}+\mathrm{jets}$ differential cross section as a function of $\Delta \phi_{\mathrm{Z}, \text { Jet } 1}$ in different $p_{\mathrm{T}}(\mathrm{Z})$ region: $p_{\mathrm{T}}(\mathrm{Z})<10 \mathrm{GeV}, 30 \leq p_{\mathrm{T}}(\mathrm{Z})<50 \mathrm{GeV}$ and $p_{\mathrm{T}}(\mathrm{Z}) \geq 100 \mathrm{GeV}[10]$.

and $106 \mathrm{GeV}$ with two leptons with $|\eta|<2.4$, $p_{\mathrm{T}}^{\text {leading lep }}>25 \mathrm{GeV}$, $p_{\mathrm{T}}^{\text {sub-leading }}>20 \mathrm{GeV}$. Jets are required to have $p_{\mathrm{T}}>30 \mathrm{GeV},|\eta|<2.4$ and a spatial separation from the dressed leptons of $R>0.4$.

Fig 3 shows the differential cross section as a function of the azimuthal correlation between the $\mathrm{Z}$ boson and the leading jet, $\Delta \phi_{\mathrm{Z}, \text { Jet } 1}$, in three different $p_{\mathrm{T}}(\mathrm{Z})$ bins: $p_{\mathrm{T}}(\mathrm{Z})<10 \mathrm{GeV}, 30 \leq p_{\mathrm{T}}(\mathrm{Z})<50$ $\mathrm{GeV}$ and $p_{\mathrm{T}}(\mathrm{Z}) \geq 100 \mathrm{GeV}$. In the low $p_{\mathrm{T}}(\mathrm{Z})$ region $\left(p_{\mathrm{T}}(\mathrm{Z})<10 \mathrm{GeV}\right)$ the $\mathrm{Z}$ boson is only very weakly correlated with the leading jet, the distribution is almost flat. In the large $p_{\mathrm{T}}(\mathrm{Z})$ region $\left(p_{\mathrm{T}}(\mathrm{Z}) \geq 100 \mathrm{GeV}\right)$ the $\mathrm{Z}$ boson is highly correlated with the leading jet, the distribution falls over more than two orders of magnitude from the back-to-back region to the small $\Delta \phi_{\mathrm{Z}, \text { Jet } 1}$ region. The systematic uncertainty in the low $p_{\mathrm{T}}(\mathrm{Z})$ region is $O(10 \%)$. the prediction from NLO MG5_AMC with and without multiparton interactions (MPI) are compared with the measurement. In the low $p_{\mathrm{T}}(\mathrm{Z})$ region, the contribution from MPI weighs for about $40 \%$ of the total. The MPI contributes significantly in the low $p_{\mathrm{T}}(\mathrm{Z})$ regions, for $p_{\mathrm{T}}(\mathrm{Z})>100 \mathrm{GeV}$ MPI becomes negligible.

The predictions from MG5AMC+CA3 $(Z+1)$ NLO, MG5AMC+CA3 $(Z+2)$ NLO and GenevA have been compared with the measurement, as shown in fig 4 . The prediction of Geneva for $Z+j e t$ observables is similar to the ones from MG5AMC+CA3 $(Z+1)$ NLO. One can see the missing MPI contribution in MG5AMC+CA3 $(Z+1)$ NLO when compared to GENEva which includes MPI. The contribution from higher order matrix elements becomes important especially in the low $\Delta \phi_{\mathrm{Z}, \text { Jet } 1}$ region as shown by the prediction MG5AMC+CA3 $(\mathrm{Z}+2) \mathrm{NLO}$.

\section{Conclusion}

We have presented the single most precise direct measurement of the $\mathrm{Z}$ boson invisible width by the CMS experiment. The differential cross section for the production of a $\mathrm{Z}$ boson in association with jets have been measured in details by CMS. Good agreement can be achieved between data and predictions when including contributions of multiparton interactions, parton shower, parton densities as well as multijet matrix-element merging. 

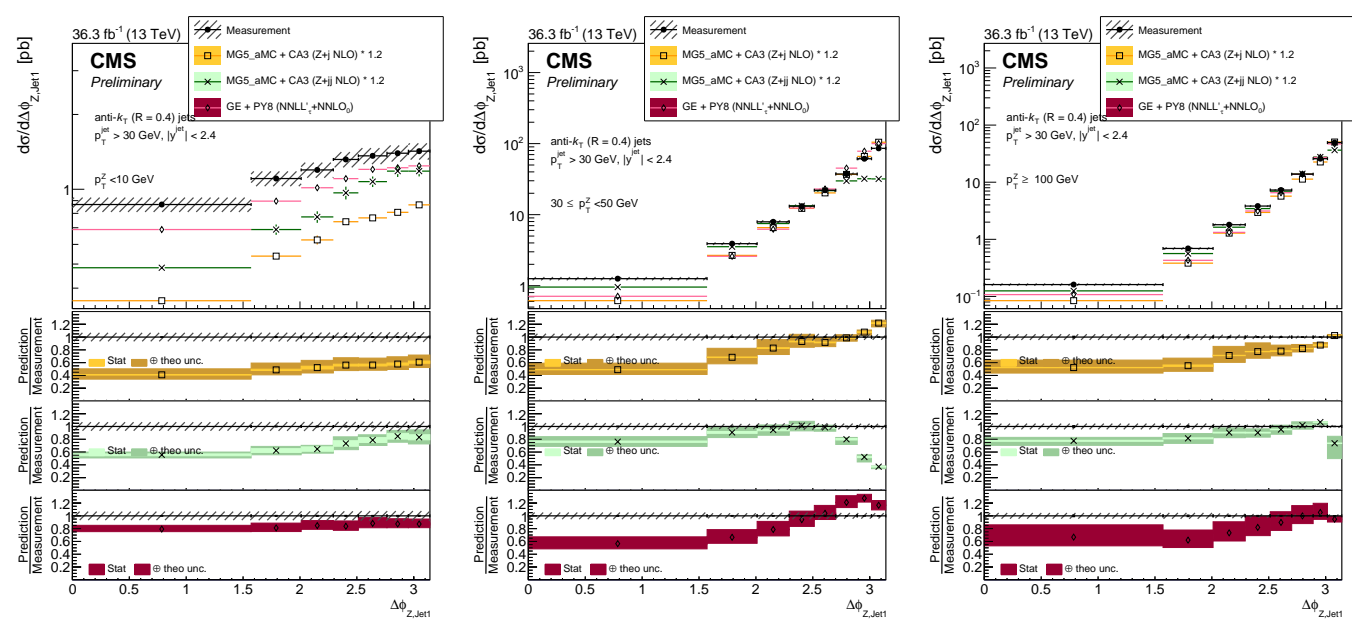

Figure 4: Measured $\mathrm{Z}+$ jets differential cross section as a function of $\Delta \phi_{\mathrm{Z}, \text { Jet } 1}$ in different $p_{\mathrm{T}}(\mathrm{Z})$ region: $p_{\mathrm{T}}(\mathrm{Z})<10 \mathrm{GeV}, 30 \leq p_{\mathrm{T}}(\mathrm{Z})<50 \mathrm{GeV}$ and $p_{\mathrm{T}}(\mathrm{Z}) \geq 100 \mathrm{GeV}[10]$.

\section{References}

[1] S. Drell and T.-M. Yan, "Massive Lepton Pair Production in Hadron-Hadron Collisions at High-Energies", Phys.Rev.Lett. 25 (1970) 316-320.

[2] CMS Collaboration, "Measurement of the transverse momentum spectra of weak vector bosons produced in proton-proton collisions at $\sqrt{s}=8 \mathrm{TeV}$ ", JHEP 02 (2017) 096, arXiv: 1606.05864.

[3] ATLAS Collaboration, "Measurement of the transverse momentum and $\phi_{\eta}^{*}$ distributions of Drell-Yan lepton pairs in proton-proton collisions at $\sqrt{s}=8 \mathrm{TeV}$ with the ATLAS detector", Eur. Phys. J. C76 (2016), no. 5, 291, arXiv: 1512.02192.

[4] CMS Collaboration, "Measurement of the rapidity and transverse momentum distributions of Z Bosons in $p p$ collisions at $\sqrt{s}=7$ TeV", Phys.Rev. D85 (2012) 032002, arXiv: 1110 4973.

[5] ATLAS collaboration, Measurement of the transverse momentum distribution of Drell-Yan lepton pairs in proton-proton collisions at $\sqrt{s}=13 \mathrm{TeV}$ with the ATLAS detector, 1912.02844.

[6] CMS collaboration, Measurements of differentialZ boson production cross sections in protonproton collisions at $\sqrt{s}=13 \mathrm{TeV}$, JHEP 12 (2019) 061 [1909.04133].

[7] CMS collaboration, Precision measurement of the $Z$ invisible width with the CMS experiment in pp collisions at $\sqrt{s}=13 \mathrm{TeV}$, [cds/2775364]

[8] CMS Collaboration, "The CMS experiment at the CERN LHC. The Compact Muon Solenoid experiment", JINST 3 S08004 (2008)

[9] CMS collaboration, Measurement of multi-differential cross sections for the production of a $Z$ boson in association with jets in proton-proton collisions at $\sqrt{s}=13 \mathrm{TeV}$, [cds/2767703]

[10] CMS collaboration, Azimuthal correlations in Z+jets events at $13 \mathrm{TeV}$, [cds/2777194] 